African American race as significant risk factors for CRI. The actuarial risks of $\mathrm{CRI}$ at 5 and 10 years were $4.0 \%$ and $11.8 \%$, respectively, and the risk of death was more than ninefold higher in those who developed this condition than in those who did not.

Original article Lee CK et al. (2007) Pre-transplant risk factors for chronic renal dysfunction after pediatric heart transplantation: a 10-year national cohort study. J Heart Lung Transplant 26: 458-465

\section{Non-aspirin NSAIDs increase the risk of iron deficiency in hemodialysis patients}

Wang et al. have investigated the relationship between the use of NSAIDs and iron status in 446 people receiving maintenance hemodialysis. NSAIDs are often used to treat bone or joint pain in this population. The authors conclude that the use of non-aspirin NSAIDs increases the risk of iron deficiency. By contrast, low-dose aspirin therapy does not have this effect.

During the 3 months preceding the index date, 95 patients were treated with aspirin, and 103 with non-aspirin NSAIDs. In comparison with those who received no anti-inflammatory therapy, patients who received non-aspirin NSAIDs-but not those who received aspirin-had significantly lower levels of serum iron $(71.9 \mu \mathrm{g} / \mathrm{dl}[12.9 \mu \mathrm{mol} / \mathrm{l}]$ vs $60.6 \mu \mathrm{g} / \mathrm{dl}$ [10.8 $\mu \mathrm{mol} / \mathrm{l}] ; P=0.002)$ and transferrin saturation $(28.5 \%$ vs $24.6 \% ; P=0.011)$. These differences were observed despite a higher proportion of patients in the non-aspirin NSAID group having received iron preparations. The proportions of patients who received recombinant human erythropoietin or who had positive fecal blood tests did not differ between study groups. Logistic regression analysis revealed that non-aspirin NSAID therapy was associated with a twofold increased risk of requiring iron supplementation.

The authors suggest that, when compared with non-aspirin NSAIDS, the use of aspirin in hemodialysis patients could be associated with lower risks of occult bleeding and iron deficiency. They note, however, that in this study aspirin was used in low doses, as an anticoagulant but not as an analgesic.

Original article Wang X et al. (2007) Iron status and the use of non-steroidal anti-inflammatory drugs in hemodialysis patients. Ther Apher Dial 11: 215-219

\section{Hematocrit targets at for-profit US dialysis facilities are higher than recommended}

Epoetin for anemia management is one of the most costly components of caring for patients with end-stage renal disease. Using the US Renal Data System Medicare claims database, Thamer et al. examined the association between dialysis facility ownership and epoetin dosing in 159,522 patients who received maintenance hemodialysis at 3,982 dialysis centers during a 2-month period in 2004.

The average adjusted dose of epoetin administered was $3,486 \mathrm{U} /$ week greater in for-profit facilities than in nonprofit facilities. At the time of this study, the hematocrit level recommended by the National Kidney Foundation-Kidney Disease Outcome Quality Initiative was 33-36\% (hemoglobin $11-12 \mathrm{mg} / \mathrm{dl}$ [6.8-7.4 mmol/l]). Patients with a hematocrit level of less than $33 \%$ being treated at for-profit facilities received larger epoetin dose increases than did patients with a similarly low hemoglobin level being treated at nonprofit facilities, with the result that fewer patients being treated at for-profit facilities had hematocrit levels below 33\%.

Notably, the proportion of patients with hematocrit levels that exceeded the recommended range was higher at for-profit facilities than nonprofit facilities ( $54 \%$ vs $47 \%)$. Indeed, patients receiving dialysis at for-profit centers had a mean hematocrit level of $36.2 \%$; the mean at nonprofit centers was $35.6 \%$. The authors conclude that financial incentives and pressure to meet clinical performance targets might encourage dialysis facility staff-particularly those at for-profit centers, at which approximately $80 \%$ of US end-stage renal disease patients are treated-to set hematocrit goals that exceed those recommended by clinical guidelines.

Original article Thamer M et al. (2007) Dialysis facility ownership and epoetin dosing in patients receiving hemodialysis. JAMA 297: 1667-1674

\section{Tacrolimus monotherapy shows efficacy in membranous nephropathy}

Some cases of membranous nephropathy resolve spontaneously, but others result in progressive renal insufficiency. Ciclosporin has been shown to slow progression of 